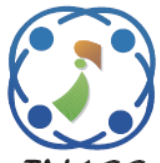

\title{
Ring Toss Game-Based Optimization Algorithm for Solving Various Optimization Problems
}

\author{
Sajjad Amiri Doumari ${ }^{1}$ \\ Hadi Givi \\ Mohammad Dehghani ${ }^{3 *}$ \\ Om Parkash Malik ${ }^{4}$ \\ ${ }^{1}$ Department of Mathematics and Computer Science, Sirjan University of Technology, Sirjan, Kerman, I. R. Iran \\ ${ }^{2}$ Department of Electrical Engineering, Shahreza Campus, University of Isfahan, Iran \\ ${ }^{3}$ Department of Electrical and Electronics Engineering, Shiraz University of Technology, Shiraz, Iran \\ ${ }^{4}$ Department of Electrical Engineering, University of Calgary, Calgary Alberta Canada \\ * Corresponding author's Email: adanbax@gmail.com
}

\begin{abstract}
There are many optimization problems in different scientific disciplines that should be solved and optimized using appropriate techniques. Population-based optimization algorithms are one of the most widely used techniques to solve optimization problems. This paper is focused on presenting a new population-based optimization approach called Ring Toss Game-Based Optimization (RTGBO) algorithm. The main idea of RTGBO is to simulate the behaviour of players and rules of the ring toss game in the design of the proposed algorithm. The main feature of the proposed RTGBO algorithm is the lack of control parameters. Steps of implementing RTGBO are described in detail and the proposed algorithm is mathematically modeled. The ability of RTGBO to solve optimization problems is evaluated on a set of twenty-three standard objective functions. These functions are selected from three different groups including unimodal, high-dimensional multimodal, and fixed-dimensional multimodal. The performance of RTGBO is also compared with eight other well-known optimization algorithms including Genetic Algorithm (GA), Particle Swarm Optimization (PSO), Gravitational Search Algorithm (GSA), Teaching Learning-Based Optimization (TLBO), Gray Wolf Optimizer (GWO), Emperor Penguin Optimizer (EPO), Hide Objects Game Optimization (HOGO), and Shell Game Optimization (SGO). The results of optimization of objective functions of unimodal type indicate the high exploitation ability of RTGBO in solving optimization problems. On the other hand, the results of optimizing the multi-model type objective functions indicate the acceptable exploration ability of RTGBO. The results also confirm the superiority of the proposed RTGBO algorithm over mentioned optimization techniques.
\end{abstract}

Keywords: Optimization, Population-based optimization, Game-based, Ring toss game, Ring toss game-based optimization, Optimization problems.

\section{Introduction}

Optimization is a science, which aims to find the best solution for a specific problem that has several different solutions according to the conditions and limitations. Each optimization problem has three main parts: variables, constraints, and problem objectives. Considering different parts of an optimization problem, it must be modeled mathematically. After mathematical modeling, the optimization problem should be optimized using an appropriate method. Population-based optimization algorithms are one of the most widely used and efficient methods in solving various optimization problems [1].
Population-based optimization algorithms are inspired by natural processes, physical phenomena, game rules, and the behavior of living things such as animals, plants, and humans. One important point about the optimization algorithms is that there is no need to derivative operators and their performance is only based on random search. Therefore, populationbased optimization algorithms refer to those methods that can provide suitable solutions to optimization problems based on random scan of the search space [2].

Each optimization problem has a basic solution called global optimum. On the other hand, different solutions provided by optimization algorithms are not necessarily the global optimum. In fact, these 
solutions are very close to the global optimum, even if they are not exactly the global optimal solution. For this reason, the solutions obtained by optimization algorithms are called quasi-optimal solutions [3]. Numerous optimization algorithms have been developed and improved by scientists with the aim of providing more appropriate quasi-optimal solutions, which are closer to the global optimum. In this regard, optimization algorithms have been applied by scientists in various fields such as energy [4, 5], protection [6], Energy Commitment (EC) [7, 8], electrical engineering [9-13], and energy carriers [14, $15]$ to achieve the optimal solution.

The contribution of this paper is designing a new population-based optimization algorithm entitled Ring Toss Game-Based Optimization (RTGBO) algorithm to solve various optimization problems in different disciplines of science and engineering. The population members in RTGBO are the game rings that are thrown towards the bars. The main idea in designing TRGBO is to simulate the ring toss game, in which game rings are thrown towards the score bars The main features of RTGBO are simplicity of equations and easy implementation on optimization problems as well as the lack of any control parameters. RTGBO is mathematically modeled and then implemented on a set of twenty-three standard objective functions of three different types to evaluate its capability.

The rest of the article is organized in such a way that in Section 2, a literature survey on optimization algorithms is presented. The proposed RTGBO algorithm is introduced and modelled in Section 3. Then the simulation results and analysis are presented in Section 4. Finally, Section 5 provides conclusions as well as some suggestions for future investigations.

\section{Background}

As mentioned so far, many optimization algorithms based on different ideas have been developed in different sciences for solving various optimization problems. Although the idea of designing optimization algorithms is different, all of these algorithms provide a solution to the problem based on a random search in the problem-solving space. Therefore, the main criterion for the superiority of optimization algorithms over each other is to provide the best quasi-optimal solutions. This has been the main reason for the design of many optimization algorithms by researchers. In this section, optimization algorithms from the perspective of design idea are studied. Population-based optimization algorithms are classified into four different groups including physics-based, swarm- based, evolutionary-based, and game-based optimization algorithms based on the design idea.

Physics-based optimization algorithms are inspired by various laws and processes of physics. Simulated Annealing (SA) is one of the optimization algorithms of this group, which was inspired by the annealing process [16]. Gravitational Search Algorithm (GSA) is another physics-based optimization algorithm, which was presented based on the simulation of the law of gravity between objects [17]. Mathematical modelling of the momentum law and Newtonian laws of motion were used in the design of the Momentum Search Algorithm (MSA) [18]. The Spring Search Algorithm (SSA) was described based on the mathematical modeling of Hooke's law in a system consisting of weights and springs [19]. Some other popular physics-based optimization algorithms are: Curved Space Optimization (CSO) [20], Central Force Optimization (CFO) [21], Galaxy-based Search Algorithm (GbSA) [22], Big-Bang Big-Crunch (BBBC) [23], Henry Gas Solubility Optimization (HGSO) [24], Binary Spring Search Algorithm (BSSA) [1, 25], Electromagnetic Field Optimization (EFO) [26], and Charged System Search (CSS) [27].

Swarm-based optimization algorithms are presented based on simulating swarm behaviour of living organisms and other natural processes. Particle Swarm Optimization (PSO) is one of the most famous algorithms in this group, which is based on mathematical modeling of bird swarm motion [28]. Ant Colony Optimization (ACO) algorithm is another widely used algorithm in this category, which is based on simulating the behavior of ants in finding the shortest path to reach food source [29, 30]. In designing the Gray Wolf Optimizer (GWO), the leadership hierarchy and the mechanism of hunting gray wolves in nature are imitated [31]. Mathematical modeling of the patient treatment process followed by the doctor was applied in the design of the "Doctor and Patient" Optimization (DPO) algorithm [8]. Some of the other swarm-based optimization algorithms are: Firefly Algorithm (FA) [32], Seagull Optimization Algorithm (SOA) [33], Multi Leader Optimizer (MLO) [34], Grasshopper Optimization Algorithm (GOA) [35], Whale Optimization Algorithm (WOA) [36], Artificial Bee Colony (ABC) [37], Group Optimization (GO) [38], Tunicate Swarm Algorithm (TSA) [39], Following Optimization Algorithm (FOA) [40], Emperor Penguin Optimizer (EPO) [41], Donkey Theorem Optimization (DTO) [42], Rat Swarm Optimizer (RSO) [43], and "The Good, the Bad, and the Ugly" Optimizer (GBUO) [44].

Evolutionary-based optimization algorithms are inspired by genetic science and the reproductive process. Genetic Algorithm (GA) is one of the most 
famous and widely used algorithms in this group. The main idea in designing GA is to model the reproductive process as well as Darwin's theory of evolution [45]. Artificial Immune System (AIS) algorithm is another evolutionary-based optimization technique, which is designed based on the simulation of the human body's defence system [46]. Some other evolutionary-based optimization algorithms are: Evolution Strategy (ES) [47], Biogeography-Based Optimizer (BBO) [48], Evolutionary Programming (EP) [49] ,Genetic Programming (GP) [50], Improved Quantum-Inspired Differential Evolution (IQDE) algorithm [51], and Differential Evolution (DE) [52].

Game-based optimization algorithms are inspired by the rules of various games as well as the behaviour of players in these games. Mathematical modeling of the behavior of players and clubs in the football game league was used in the design of the Football GameBased Optimization (FGBO) [53]. Orientation Search Algorithm (OSA) was proposed based on mathematical modeling of the behavior of players in the orientation game, who move according to the direction of the referee's hand on the playing field [54]. Some of the other game-based optimization algorithms are: Hide Objects Game Optimization (HOGO) [55], Darts Game Optimizer (DGO) [56], Shell Game Optimization (SGO) [57], Dice Game Optimizer (DGO) [58], and Binary Orientation Search Algorithm (BOSA) [59].

\section{Ring toss game-based optimization}

In this section, the theory and various steps of the RTGBO algorithm are described and then the mathematical modelling process of the proposed algorithm is presented.

\subsection{Theory of RTGBO}

RTGBO is a population-based optimization algorithm, which belongs to game-based category. RTGBO is designed based on simulating the rules of ring toss game and the behaviour of players in throwing rings towards the scoring bar. In RTGBO, population members are rings that are initially randomly placed on the playground. These rings are thrown in the direction of one of the score bars in a random throw. These throws are continued in a repetitive process until the end of the game. From the viewpoint of optimization algorithms, the position of each ring on the playground in the proposed RTGBO algorithm is a solution to the optimization problem. The objective function is evaluated based on each of these situations. Therefore, appropriate positions of the playground, which is in fact the search space of the problem, are determined. In the RTGBO, it is assumed that the score bars are installed in areas that result in better values for the objective function. Then, in the next iteration, the rings are thrown again towards the score bars installed in the new position. This iterative process is repeated until the end of the algorithm iterations or achieving the appropriate solution to the optimization problem.

\subsection{Mathematical modeling of RTGBO algorithm}

In this part, the RTGBO algorithm is mathematically modeled to be implemented on optimization problems. The values of the optimization problem variables are determined based on the position of each population member in the search space. Thus, each member of the population is a vector with the number of elements equal to the number of problem variables.

The population members of the RTGBO algorithm are represented by a matrix called the population matrix as expressed in Eq. (1).

$$
X=\left[\begin{array}{c}
X_{1} \\
\vdots \\
X_{i} \\
\vdots \\
X_{N}
\end{array}\right]_{N \times m}=\left[\begin{array}{ccccc}
x_{1,1} & \cdots & x_{1, d} & \cdots & x_{1, m} \\
\vdots & \ddots & \vdots & \ddots & \vdots \\
x_{i, 1} & \cdots & x_{i, d} & \cdots & x_{i, m} \\
\vdots & \ddots & \vdots & \ddots & \vdots \\
x_{N, 1} & \cdots & x_{N, d} & \cdots & x_{N, m}
\end{array}\right]_{N \times m}
$$

Here, $X$ is the population matrix, $X_{i}$ represents the $i$ 'th population member, $x_{i, d}$ is the value of $d^{\prime}$ th variable for $i$ 'th population member, $N$ is the number of population members, and $m$ is the number of problem variables.

The objective function is evaluated based on the values of the population matrix and the results can be displayed as a vector using Eq. (2).

$$
O F=\left[\begin{array}{lllll}
O F_{1} & \ldots & O F_{i} & \ldots & O F_{N}
\end{array}\right]_{1 \times N}
$$

Here, $O F$ is the vector of the objective function and $O F_{i}$ denotes the objective function value for $i$ 'th population member.

At this stage of the modeling, score bars are installed in appropriate locations of the search space, where population members provide better values for the objective function using Eq. (3).

$$
S B=\left[\begin{array}{c}
S B_{1} \\
\vdots \\
S B_{i} \\
\vdots \\
S B_{N_{S B}}
\end{array}\right]_{N_{S B} \times m}
$$


Here, $S B$ is the matrix of locations of the score bars, $S B_{i}$ indicates the position of $i$ 'th score bar in the search space, and $N_{S B}$ is the number of score bars, which is equal to $10 \%$ of the population members.

In the next step, throwing of the rings towards the score bars is modeled. Each of the rings is randomly thrown towards one of the bars. This step of the RTGBO and calculation of the new positions of the rings are accomplished using Eq. (4) to (7).

$$
\begin{gathered}
F=\text { round }(1+r) \\
d x_{i, d}= \begin{cases}r\left(s b_{i, d}-F x_{i, d}\right), & O F_{S B_{i}}<O F_{i} \\
r\left(x_{i, d}-F s b_{i, d}\right), & \text { else }\end{cases} \\
x_{i, d}^{\text {new }}=x_{i, d}+d x_{i, d} \\
X_{i}=\left\{\begin{array}{cl}
X_{i}^{\text {new }}, & O F_{i}^{\text {new }}<O F_{i} \\
X_{i}, & \text { else }
\end{array}\right.
\end{gathered}
$$

Here, $d x_{i, d}$ is the value of displacement for $i^{\prime}$ th population member in $d^{\prime}$ th dimension, $s b_{i, d}$ is the $d^{\prime}$ 'th dimension of the score bar position, $O F_{S B_{i}}$ is the value of the objective function of $i$ th score bar, $x_{i, d}^{\text {new }}$ is the new suggested position for $i$ th population member in $d$ 'th dimension, $O F_{i}^{\text {new }}$ is the value of the objective function for new suggested position of $i$ 'th population member, and $r$ is a random number in [0 1] interval.

Update of the population members is repeated according to Eqs. (2) to (7) until the stop condition is reached. After completing the iterations of the algorithm, the most suitable quasi-optimal solution obtained by the proposed algorithm is available. The implementation steps of the proposed RTGBO algorithm are shown as a flowchart in Fig. 1.

\section{Simulation studies and discussion}

In this section, the ability of the proposed RTGBO algorithm to solve optimization problems and provide appropriate quasi-optimal solutions is evaluated. For this purpose, the RTGBO is implemented on a set of twenty-three standard objective functions of three different types including unimodal, high-dimensional multimodal, and fixed-dimensional multimodal functions. These objective functions have been adapted from [31]. Also, the optimization results obtained by the RTGBO algorithm are compared with eight other optimization algorithms including Genetic Algorithm (GA), Particle Swarm Optimization (PSO), Gravitational Search Algorithm (GSA), Teaching Learning-Based Optimization (TLBO), Gray Wolf
Optimizer (GWO), Emperor Penguin Optimizer (EPO), Hide Objects Game Optimization (HOGO), and Shell Game Optimization (SGO). Average (Ave) and standard deviation (std) of the best quasi-optimal solutions are considered as the comparison criterion.

\subsection{Evaluation for unimodal objective functions}

Seven objective functions F1 to F7 are considered in order to analyze the ability of the RTGBO to provide a quasi-optimal solution for objective functions of unimodal type. The optimization results for these objective functions using RTGBO and eight other optimization algorithms are presented in Table 1. The simulation results show the superiority of the RTGBO over the other eight optimization algorithms.

\subsection{Evaluation for high-dimensional multimodal objective functions}

Six objective functions F8 to F13 are selected from high-dimensional multimodal category. The results of implementing RTGBO and eight other optimization algorithms to provide quasi-optimal solution are presented in Table 2. Simulation results indicate acceptable ability of the proposed algorithm to optimize this type of optimization problems.

\subsection{Evaluation for fixed-dimensional multimodal objective functions}

Ten objective functions F14 to F23 are selected from fixed-dimensional multimodal functions in order to analyze the performance of the RTGBO in solving fixed-dimensional multimodal optimization problems. The performance results of the RTGBO and eight other optimization algorithms in solving this type of objective functions are presented in Table 3. The optimization results show the optimal performance of the RTGBO in solving fixed-dimensional optimization problems.

Also, comparing the results obtained from the RTGBO with other optimization algorithms shows that the proposed RTGBO algorithm is more competitive than other eight optimization algorithms.

\subsection{Discussion and theoretical explanation}

exploitation and exploration are two very important and key indicators in evaluating and comparing the performance of optimization algorithms. 

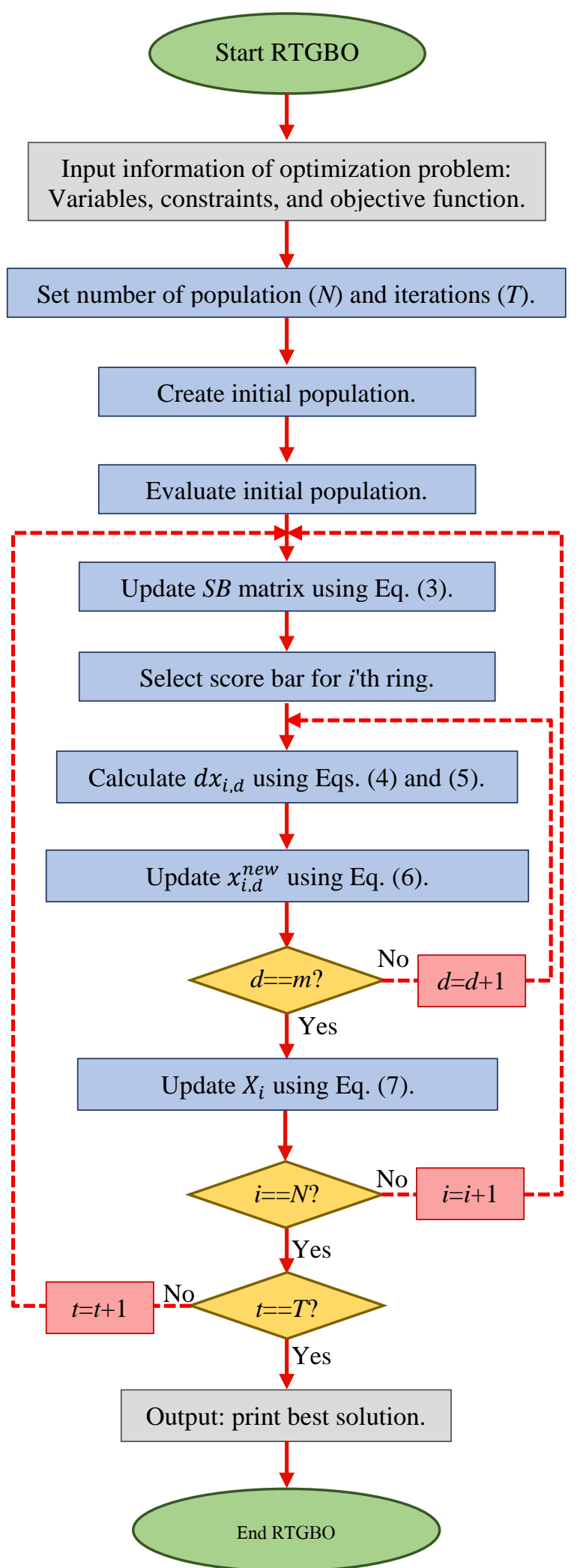

Figure. 1 Flowchart of RTGBO algorithm

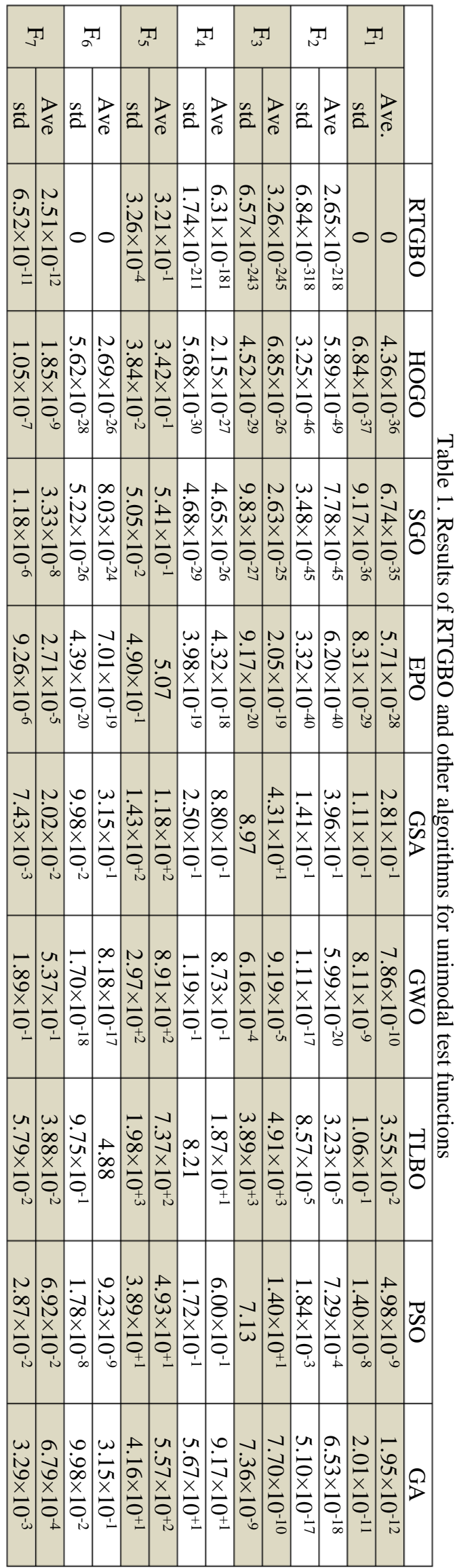




\begin{tabular}{|c|c|c|c|c|c|c|c|c|c|c|c|c|}
\hline 3 & 7 & $\frac{T}{N}$ & & $\exists$ & & $\frac{7}{0}$ & & T & & $T_{\infty}^{7}$ & & \\
\hline$\stackrel{n}{2}$ & 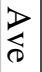 & $\frac{n}{2}$ & 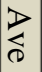 & $\frac{n}{a}$ & 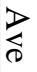 & $\frac{n}{2}$ & $\underset{<}{\mathbb{2}}$ & $\frac{n}{a}$ & $\frac{1}{4}$ & $\frac{n}{2}$ & 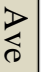 & \\
\hline $\begin{array}{l}\circ \\
8 \\
8\end{array}$ & $\stackrel{\circ}{\circ}$ & 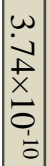 & $\begin{array}{l}\bar{\omega} \\
\tilde{N} \\
x \\
\vdots \\
0 \\
\dot{b}\end{array}$ & 0 & 0 & 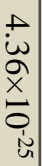 & 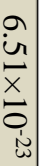 & 0 & 0 & $\begin{array}{l}\frac{\vec{u}}{x} \\
\frac{\vec{x}}{0}\end{array}$ & $\begin{array}{c}1 \\
\dot{N} \\
x \\
\dot{x} \\
\vdots \\
\pm\end{array}$ & 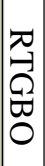 \\
\hline
\end{tabular}

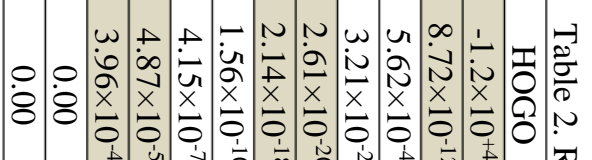

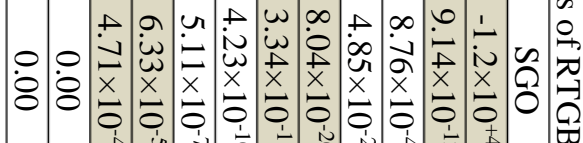

$N-\omega$ u

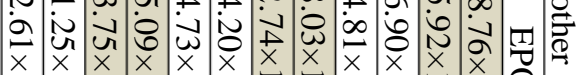

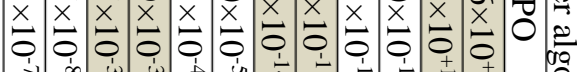

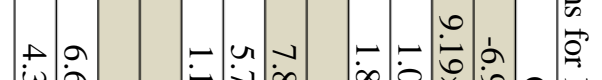

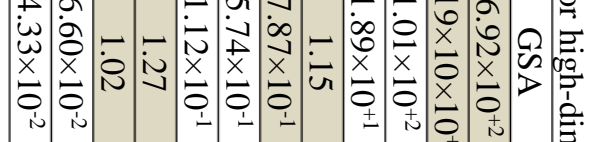

N.

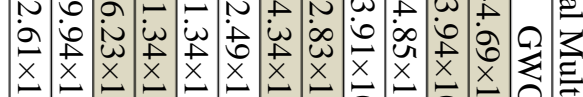

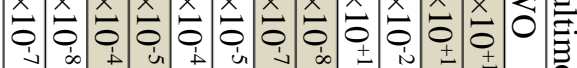

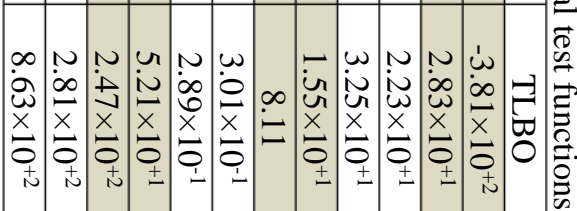

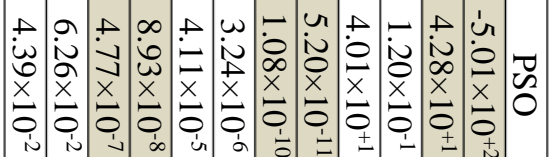

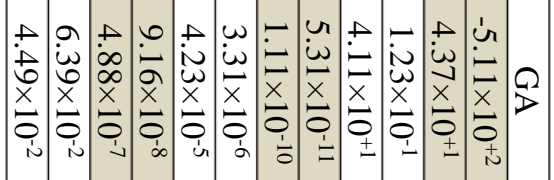

\begin{tabular}{|c|c|c|c|c|c|c|c|c|c|}
\hline 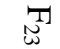 & $\underset{N}{N}$ & $\underset{N}{T}$ & $\mathbb{N}_{0}^{\top}$ & $\frac{\pi}{6}$ & $\underset{\infty}{T}$ & $\frac{T}{3}$ & $\frac{\pi}{a}$ & $\frac{\pi}{u}$ & $\frac{T}{ \pm}$ \\
\hline
\end{tabular}

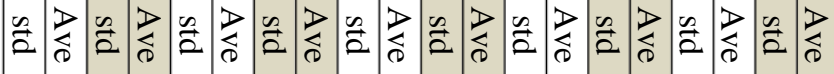

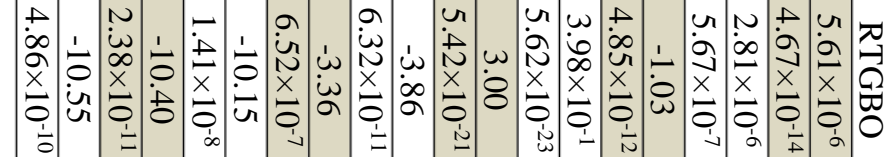

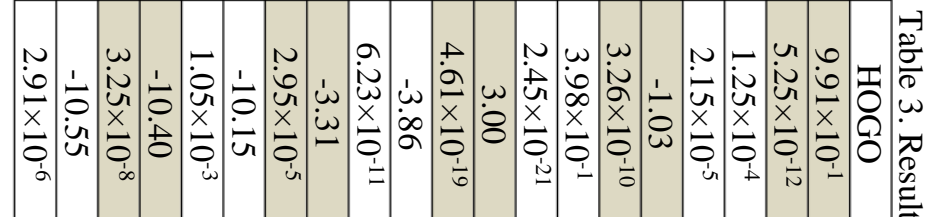

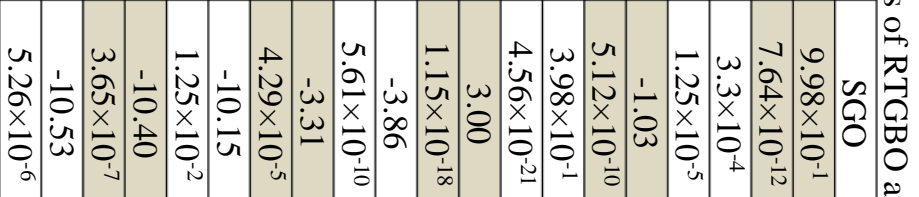

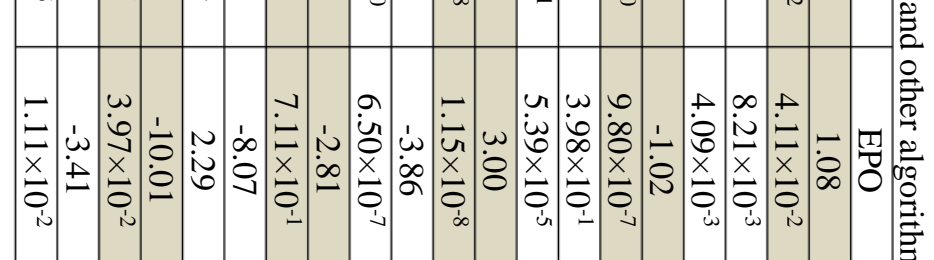

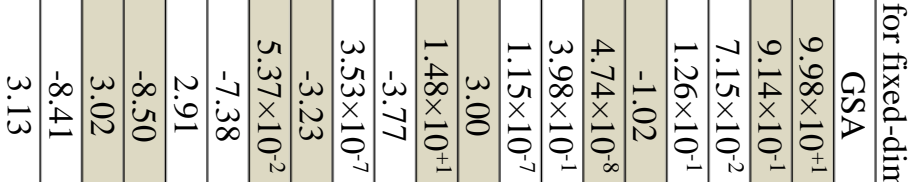

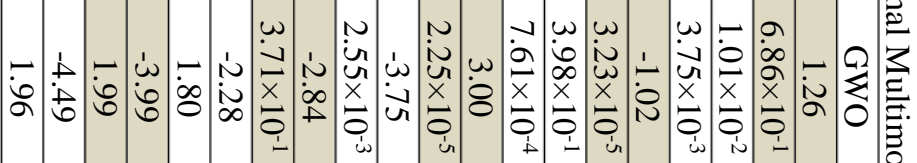

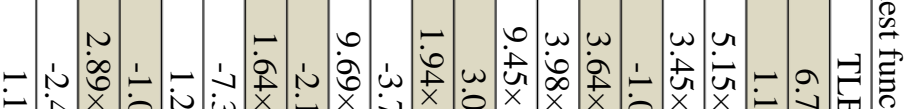

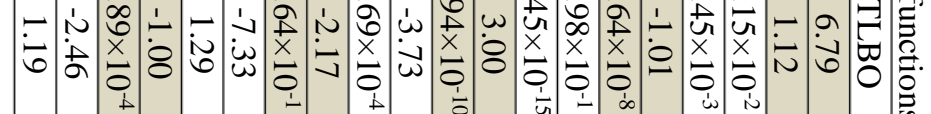

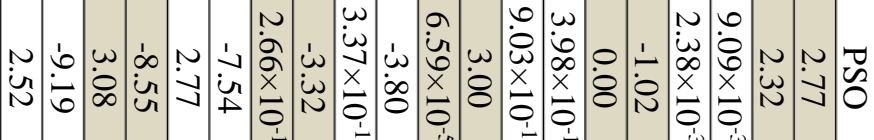

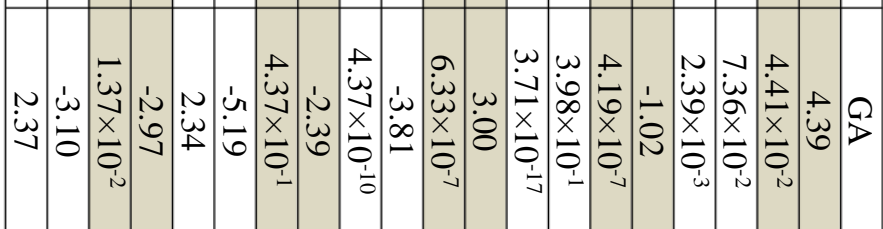


Exploitation means the ability of an optimizationalgorithm to provide a suitable quasioptimal solution as well as close to the global optimal. Therefore, in the analysis of optimization algorithms, the algorithm that can provide the most suitable quasi-optimal solution has a higher exploitation power. The F1 to F7 objective functions have only one main optimal solution and are therefore very suitable for evaluating the exploitation index. The results of the RTGBO operation and the other eight algorithms are presented in Table 1. Based on the results in this table, RTGBO has provided global optimizations for F1 and F6, as well as much bettersuited quasi-optimal solutions for F2, F3, F4, F5, and F7 than other optimization algorithms. Analysis of the optimization results of the proposed algorithm and its comparison with competing algorithms indicates the acceptable and high exploitation ability of RTGBO.

Exploration means the ability of an optimization algorithm to accurately search the search space. An optimization algorithm must be able to scan different areas of the search space and be able to bypass local optimized areas. This indicator is especially important for solving optimization problems with several optimal local solutions. The F8 to F23 objective functions, in addition to the global optimal solution have also several quasi-optimal solutions. The F8 to F23 objective functions, in addition to the global optimal solution have also several quasioptimal solutions and are therefore very suitable for evaluating the exploration index. The results of the exploration power evaluation of different optimization algorithms are presented in Tables 2 and 3. Based on the analysis of the results of these tables, RTGBO has provided the optimal global solution for F8, F9, F11, F13, F6, F17, F18, F19, F20, F21, F22, and F23 by overcoming local optimal solutions. RTGBO has also shown good performance in optimizing other multimodal objective functions. The performance analysis of the proposed algorithm in the objective functions with local optimal solutions indicates the acceptable exploration ability of RTGBO in accurate search of the search space.

\section{Conclusions and feature works}

In this paper, a new game-based optimization algorithm entitled Ring Toss Game-Based Optimization (RTGBO) algorithm was presented for solving various optimization problems. The main idea of the proposed RTGBO algorithm was inspired by the ring toss game. RTGBO was mathematically modelled and then applied on twenty-three standard objective functions with the aim of providing suitable quasi-optimal solutions. Unimodal objective functions are used to evaluate the exploitation ability of optimization algorithms. High-dimensional multimodal, and fixed-dimensional multimodal objective functions are applied in order to evaluate exploration ability of optimization algorithms. Based on the results of implementing the RTGBO on standard objective functions, it is determined that the RTGBO has a high ability to search for problemsolving space as an exploration index and to achieve a quasi-optimal solution very close to the global optimal as an exploitation index.

For further analysis, the results obtained by the RTGBO algorithm were compared with eight other well-known optimization algorithms including GA, PSO, GSA, TLBO, GWO, EPO, HOGO, and SGO. The simulation results showed the desirable performance of the RTGBO for solving various optimization problems and also the superiority of the proposed algorithm over the other eight optimization algorithms.

The authors suggest some ideas and perspectives for future studies. Design of the binary version as well as multi-objective version of RTGBO is an interesting topic for future investigations. Moreover, implementing RTGBO algorithm on various optimization problems and real-world optimization problems could achieve some significant contributions, as well.

\section{Conflicts of Interest}

The authors declare no conflict of interest.

\section{Author Contributions}

Conceptualization, S.A.D and H.G.; methodology, M.D.; software, S.A.D. and M.D.; validation, H.G., O.P.M., and M.D.; formal analysis, O.P.M. and H.G.; investigation, M.D., S.A.D., and O.P.M.; resources, M.D.; data curation, H.G.; writing - original draft preparation, S.A.D.; writing-review and editing, O.P.M. and H.G.; visualization, M.D; supervision, M.D.; project administration, S.A.D.; funding acquisition, O.P.M.

\section{References}

[1] M. Dehghani, Z. Montazeri, A. Dehghani, O. P. Malik, R. Morales-Menendez, G. Dhiman, N. Nouri, A. Ehsanifar, J. M. Guerrero, and R. A. Ramirez-Mendoza, "Binary Spring Search Algorithm for Solving Various Optimization Problems", Applied Sciences, Vol. 11, No. 3, pp. 1286, 2021. 
[2] M. Dehghani, Z. Montazeri, A. Dehghani, H. Samet, C. Sotelo, D. Sotelo, A. Ehsanifar, O. P. Malik, J. M. Guerrero, and G. Dhiman, "DM: Dehghani Method for modifying optimization algorithms", Applied Sciences, Vol. 10, No. 21, pp. 7683, 2020.

[3] M. Dehghani, Z. Montazeri, G. Dhiman, O. Malik, R. Morales-Menendez, R. A. RamirezMendoza, A. Dehghani, J. M. Guerrero, and L. Parra-Arroyo, "A spring search algorithm applied to engineering optimization problems", Applied Sciences, Vol. 10, No. 18, pp. 6173, 2020.

[4] M. Dehghani, Z. Montazeri, and O. P. Malik, "Energy Commitment: A Planning of Energy Carrier Based on Energy Consumption", Electrical Engineering \& Electromechanics, No. 4, pp. 69-72, 2019.

[5] M. Dehghani, M. Mardaneh, O. P. Malik, J. M. Guerrero, C. Sotelo, D. Sotelo, M. Nazari-Heris, K. Al-Haddad, and R. A. Ramirez-Mendoza, "Genetic Algorithm for Energy Commitment in a Power System Supplied by Multiple Energy Carriers", Sustainability, Vol. 12, No. 23, pp. 10053, 2020.

[6] A. Ehsanifar, M. Dehghani, and M. Allahbakhshi, "Calculating The Leakage Inductance for Transformer Inter-Turn Fault Detection Using Finite Element Method", In: Proc. of Iranian Conference on Electrical Engineering (ICEE), Tehran, Iran, pp.13721377, 2017.

[7] M. Dehghani, M. Mardaneh, O. P. Malik, J. M. Guerrero, R. Morales-Menendez, R. A. Ramirez-Mendoza, J. Matas, and A. Abusorrah, "Energy Commitment for a Power System Supplied by Multiple Energy Carriers System using Following Optimization Algorithm", Applied Sciences, Vol. 10, No. 17, pp. 5862, 2020.

[8] M. Dehghani, M. Mardaneh, J. M. Guerrero, O. P. Malik, R. A. Ramirez-Mendoza, J. Matas, J. C. Vasquez, and L. Parra-Arroyo, "A new "Doctor and Patient" optimization algorithm: An application to energy commitment problem", Applied Sciences, Vol. 10, No. 17, pp. 5791, 2020.

[9] M. Dehghani, Z. Montazeri, and O. Malik, "Optimal sizing and placement of capacitor banks and distributed generation in distribution systems using spring search algorithm", International Journal of Emerging Electric Power Systems, Vol. 21, No. 1, 2020.

[10] M. Dehghani, Z. Montazeri, O. P. Malik, K. AlHaddad, J. M. Guerrero, and G. Dhiman, "A
New Methodology Called Dice Game Optimizer for Capacitor Placement in Distribution Systems", Electrical Engineering \& Electromechanics, No. 1, pp. 61-64, 2020.

[11] S. Dehbozorgi, A. Ehsanifar, Z. Montazeri, M. Dehghani, and A. Seifi, "Line Loss Reduction and Voltage Profile Improvement in Radial Distribution Networks Using Battery Energy Storage System", In: Proc. of IEEE 4th International Conference on Knowledge-Based Engineering and Innovation (KBEI), Tehran, Iran, pp.0215-0219, 2017.

[12] Z. Montazeri, and T. Niknam, "Optimal utilization of electrical energy from power plants based on final energy consumption using gravitational search algorithm", Electrical Engineering \& Electromechanics, No. 4, pp. 7073, 2018.

[13] M. Dehghani, M. Mardaneh, Z. Montazeri, A. Ehsanifar, M. J. Ebadi, and O. M. Grechko, "Spring search algorithm for simultaneous placement of distributed generation and capacitors", Electrical Engineering \& Electromechanics, No. 6, pp. 68-73, 2018.

[14] M. Dehghani, Z. Montazeri, A. Ehsanifar, A. R. Seifi, M. J. Ebadi, and O. M. Grechko, "Planning of energy carriers based on final energy consumption using dynamic programming and particle swarm optimization", Electrical Engineering \& Electromechanics, No. 5, pp. 62-71, 2018.

[15] Z. Montazeri and T. Niknam, "Energy Carriers Management Based on Energy Consumption", In: Proc. of IEEE 4th International Conference on Knowledge-Based Engineering and Innovation (KBEI)", Tehran, Iran, pp.0539-0543, 2017.

[16] P. J. Van Laarhoven and E. H. Aarts, "Simulated annealing", Simulated annealing: Theory and applications, ed: Springer, pp. 7-15, 1987.

[17] E. Rashedi, H. Nezamabadi-Pour, and S. Saryazdi, "GSA: a gravitational search algorithm", Information sciences, Vol. 179, No. 13, pp. 2232-2248, 2009.

[18] M. Dehghani, and H. Samet, "Momentum search algorithm: A new meta-heuristic optimization algorithm inspired by momentum conservation law", SN Applied Sciences, Vol. 2, No. 10, pp. 1-15, 2020.

[19] M. Dehghani, Z. Montazeri, A. Dehghani, and A. Seifi, "Spring Search Algorithm: A New MetaHeuristic Optimization Algorithm Inspired by Hooke's Law", In: Proc. of IEEE 4th International Conference on Knowledge-Based 
Engineering and Innovation (KBEI), Tehran, Iran, pp.0210-0214, 2017.

[20] F. F. Moghaddam, R. F. Moghaddam, and M. Cheriet, "Curved space optimization: a random search based on general relativity theory", arXiv preprint arXiv:1208.2214, 2012.

[21] R. A. Formato, "Central force optimization: a new nature inspired computational framework for multidimensional search and optimization", Nature Inspired Cooperative Strategies for Optimization (NICSO 2007), ed: Springer, pp. 221-238, 2008.

[22] H. Shah-Hosseini, "Principal components analysis by the galaxy-based search algorithm: a novel metaheuristic for continuous optimisation", International Journal of Computational Science and Engineering, Vol. 6, No. 1-2, pp. 132-140, 2011.

[23] O. K. Erol, and I. Eksin, "A new optimization method: big bang-big crunch", Advances in Engineering Software, Vol. 37, No. 2, pp. 106$111,2006$.

[24] F. A. Hashim, E. H. Houssein, M. S. Mabrouk, W. Al-Atabany, and S. Mirjalili, "Henry gas solubility optimization: A novel physics-based algorithm", Future Generation Computer Systems, Vol. 101, pp. 646-667, 2019.

[25] M. Dehghani, Z. Montazeri, A. Dehghani, N. Nouri, and A. Seifi, "BSSA: Binary Spring Search Algorithm", In: Proc. of IEEE 4th International Conference on Knowledge-Based Engineering and Innovation (KBEI), Tehran, Iran, pp.0220-0224, 2017.

[26] H. Abedinpourshotorban, S. M. Shamsuddin, Z. Beheshti, and D. N. Jawawi, "Electromagnetic field optimization: a physics-inspired metaheuristic optimization algorithm", Swarm and Evolutionary Computation, Vol. 26, pp. 822, 2016.

[27] A. Kaveh and S. Talatahari, "A novel heuristic optimization method: charged system search", Acta Mechanica, Vol. 213, No. 3-4, pp. 267-289, 2010.

[28] J. Kennedy, and R. Eberhart, "Particle swarm optimization", In: Proc. of ICNN'95International Conference on Neural Networks, Vol. 4, ed: IEEE, pp. 1942-1948, 1995.

[29] M. Dorigo and T. Stützle, "Ant colony optimization: overview and recent advances", Handbook of Metaheuristics, pp. 311-351, 2019.

[30] H. Givi, M. A. Noroozi, B. Vahidi, J. S. Moghani, and M. A. V. Zand, "A Novel Approach for Optimization of Z-Matrix Building Process Using Ant Colony Algorithm", Vol. 2, No. 9, pp. 8932-8937, 2012.
[31] S. Mirjalili, S. M. Mirjalili, and A. Lewis, "Grey wolf optimizer", Advances in engineering software, Vol. 69, pp. 46-61, 2014.

[32] X.-S. Yang, "Firefly algorithm, stochastic test functions and design optimisation", arXiv preprint arXiv:1003.1409, 2010.

[33] G. Dhiman, K. K. Singh, M. Soni, A. Nagar, M. Dehghani, A. Slowik, A. Kaur, A. Sharma, E. H. Houssein, and K. Cengiz, "MOSOA: A new multi-objective seagull optimization algorithm", Expert Systems with Applications, pp. 114150, 2020.

[34] M. Dehghani, Z. Montazeri, A. Dehghani, R. R. Mendoza, H. Samet, J. M. Guerrero, and G. Dhiman, "MLO: Multi Leader Optimizer", International Journal of Intelligent Engineering and Systems, Vol. 13, No. 6, pp. 364-373, 2020.

[35] S. Saremi, S. Mirjalili, and A. Lewis, "Grasshopper optimisation algorithm: theory and application", Advances in Engineering Software, Vol. 105, pp. 30-47, 2017.

[36] S. Mirjalili, and A. Lewis, "The whale optimization algorithm", Advances in engineering software, Vol. 95, pp. 51-67, 2016.

[37] D. Karaboga and B. Basturk, "Artificial bee colony (ABC) optimization algorithm for solving constrained optimization", In: Proc. of International Fuzzy Systems Association World Congress, ed: Springer, pp. 789-798, 2007.

[38] M. Dehghani, Z. Montazeri, A. Dehghani, and O. P. Malik, "GO: Group Optimization", Gazi University Journal of Science, Vol. 33, 2020.

[39] S. Kaur, L. K. Awasthi, A. Sangal, and G. Dhiman, "Tunicate swarm algorithm: a new bioinspired based metaheuristic paradigm for global optimization", Engineering Applications of Artificial Intelligence, Vol. 90, pp. 103541, 2020.

[40] M. Dehghani, M. Mardaneh, and O. Malik, "FOA:'Following'Optimization Algorithm for solving Power engineering optimization problems", Journal of Operation and Automation in Power Engineering, Vol. 8, No. 1, pp. 57-64, 2020.

[41] G. Dhiman and V. Kumar, "Emperor Penguin Optimizer: A Bio-inspired Algorithm for Engineering Problems", Knowledge-Based Systems, 2018.

[42] M. Dehghani, M. Mardaneh, O. P. Malik, and S. M. NouraeiPour, "DTO: Donkey Theorem Optimization", In: Proc. of 27th Iranian Conference on Electrical Engineering (ICEE), Yazd, Iran, pp.1855-1859, 2019.

[43] Gaurav Dhiman, Meenakshi Garg, Atulya K Nagar, Vijay Kumar, and M. Dehghani, "A 
Novel Algorithm for Global Optimization: Rat Swarm Optimizer," Journal of Ambient Intelligence and Humanized Computing, 2020.

[44] H. Givi, M. Dehghani, Z. Montazeri, R. Morales-Menendez, R. A. Ramirez-Mendoza, and N. Nouri, "GBUO: "The Good, the Bad, and the Ugly" Optimizer", Applied Sciences, Vol. 11, No. 5, pp. 2042, 2021.

[45] A. Bose, T. Biswas and P. Kuila, "A novel genetic algorithm based scheduling for multicore systems", Smart Innovations in Communication and Computational Sciences, ed: Springer, pp. 45-54, 2019.

[46] S. A. Hofmeyr, and S. Forrest, "Architecture for an artificial immune system", Evolutionary computation, Vol. 8, No. 4, pp. 443-473, 2000.

[47] H.-G. Beyer, and H.-P. Schwefel, "Evolution strategies-A comprehensive introduction", Natural computing, Vol. 1, No. 1, pp. 3-52, 2002.

[48] D. Simon, "Biogeography-based optimization", IEEE Transactions on Evolutionary Computation, Vol. 12, No. 6, pp. 702-713, 2008.

[49] L. J. Fogel, A. J. Owens, and M. J. Walsh, "Artificial intelligence through simulated evolution," 1966.

[50] J. R. Koza, "Genetic programming as a means for programming computers by natural selection", Statistics and computing, Vol. 4, No. 2, pp. 87-112, 1994.

[51] W. Deng, H. Liu, J. Xu, H. Zhao, and Y. Song, "An improved quantum-inspired differential evolution algorithm for deep belief network", IEEE Transactions on Instrumentation and Measurement, 2020.

[52] S. Das and P. N. Suganthan, "Differential evolution: a survey of the state-of-the-art", IEEE Transactions on Evolutionary Computation, Vol. 15, No. 1, pp. 4-31, 2011.

[53] M. Dehghani, M. Mardaneh, J. M. Guerrero, O. Malik, and V. Kumar, "Football game based optimization: An application to solve energy commitment problem", International Journal of Intelligent Engineering and Systems, Vol. 13, pp. 514-523, 2020.

[54] M. Dehghani, Z. Montazeri, O. P. Malik, A. Ehsanifar, and A. Dehghani, "OSA: Orientation search algorithm", International Journal of Industrial Electronics, Control and Optimization, Vol. 2, No. 2, pp. 99-112, 2019.

[55] M. Dehghani, Z. Montazeri, S. Saremi, A. Dehghani, O. P. Malik, K. Al-Haddad, and J. M. Guerrero, "HOGO: Hide Objects Game Optimization", International Journal of Intelligent Engineering and Systems, Vol. 13, No. 4, pp. 216-225, 2020.
[56] M. Dehghani, Z. Montazeri, H. Givi, J. M. Guerrero, and G. Dhiman, "Darts game optimizer: A new optimization technique based on darts game", International Journal of Intelligent Engineering and Systems, Vol. 13, pp. 286-294, 2020.

[57] D. Mohammad, M. Zeinab, O. P. Malik, H. Givi, and J. M. Guerrero, "Shell Game Optimization: A Novel Game-Based Algorithm", International Journal of Intelligent Engineering and Systems, Vol. 13, No. 3, pp. 246-255, 2020.

[58] M. DEHGHANI, Z. MONTAZERI, and O. P. MALIK, "DGO: Dice game optimizer", Gazi University Journal of Science, Vol. 32, No. 3, pp. 871-882, 2019.

[59] M. Dehghani, Z. Montazeri, O. P. Malik, G. Dhiman, and V. Kumar, "BOSA: Binary Orientation Search Algorithm", International Journal of Innovative Technology and Exploring Engineering (IJITEE), Vol. 9, No. 1, pp. 53065310, 2019. 